\title{
Effect of delta $\alpha$-fetoprotein on the detection of liver cancer recurrence
}

\author{
Li-Yue Sun ${ }^{1,2,3 \#}$, Yuan $\mathrm{He}^{1,2,3 \#}$, Qing Liu ${ }^{1,2,3}$, Fang Wang ${ }^{1,2,3}$ \\ ${ }^{1}$ State Key Laboratory of Oncology in South China, Guangzhou, China; ${ }^{2}$ Collaborative Innovation Center for Cancer Medicine, Guangzhou, China; \\ ${ }^{3}$ Department of Molecular Diagnostics, Sun Yat-sen University Cancer Center, Guangzhou, China \\ Contributions: (I) Conception and design: LY Sun, F Wang; (II) Administrative support: LY Sun; (III) Provision of study materials or patients: Q Liu; \\ (IV) Collection and assembly of data: LY Sun, Q Liu; (V) Data analysis and interpretation: Y He; (VI) Manuscript writing: All authors; (VII) Final \\ approval of manuscript: All authors. \\ "These authors contributed equally to this work. \\ Correspondence to: Fang Wang. Department of Molecular Diagnostics, Sun Yat-sen University Cancer Center, no. 651 Dongfeng East Road, \\ Guangzhou 510060, China. Email: wangfang@sysucc.org.cn.
}

Background: We explored the ability of delta $\alpha$-fetoprotein $(\triangle \mathrm{AFP})$ to detect recurrence in patients with liver cancer treated with hepatectomy.

Methods: A total of 1,846 patients diagnosed with local liver cancer who underwent hepatectomy at Sun Yat-sen University Cancer Center were enrolled in the present study. Receiver operating characteristic curve analysis was used to determine the cutoff value of $\triangle \mathrm{AFP}$ at the last follow-up or recurrence.

Results: Recurrence occurred in $51.5 \%(950 / 1,846)$ of liver cancer patients. The cutoff value of $\triangle \mathrm{AFP}$ was $1.295 \mathrm{ng} / \mathrm{mL}$ in our model. Sensitivity in our model was higher than the normal range for AFP level for detecting recurrence $(59.8 \%$ vs. $43.8 \%$ ), but specificity was similar (98.4\% vs. $99.8 \%)$. $\triangle \mathrm{AFP}$ in preoperative AFP-positive patients (77.16\% vs. 63.28\%) and AFP-negative patients (31.20\% vs. 11.70\%) was more sensitive than normal AFP. $\triangle \mathrm{AFP}$ was superior to AFP in the early $(78.13 \%$ vs. $63.75 \%$ ) or late recurrence (56.08\% vs. $39.75 \%)$ of liver cancer. Moreover, in $18.3 \%$ of patients with recurrence (174/950), $\triangle \mathrm{AFP}$ detected recurrence earlier than computed tomography/magnetic resonance imaging by 158.33 days. $\triangle \mathrm{AFP}$ during follow-up indicated a worse prognosis after hepatectomy.

Conclusions: The cutoff value of $\triangle \mathrm{AFP}$ is more sensitive for monitoring recurrence than a normal AFP level in liver cancer patients.

Keywords: Liver cancer; hepatectomy; delta $\alpha$-fetoprotein $(\triangle \mathrm{AFP})$; recurrence

Submitted Apr 19, 2020. Accepted for publication Sep 12, 2020.

doi: $10.21037 /$ tcr-20-1874

View this article at: http://dx.doi.org/10.21037/tcr-20-1874

\section{Introduction}

Hepatectomy is the only potential curative therapy for liver cancer without distant metastasis (1); however, the high incidence of tumor recurrence and metastasis is the main reason for mortality after surgery (2). Therefore, monitoring after hepatectomy is crucial for the early detection of recurrence and the subsequent determination of whether these patients who experience recurrence are eligible for additional therapy, such as repeat hepatectomy and liver transplant (3). Patients who undergo second and third hepatectomies after tumor recurrence have a 5 -year survival rate of $60 \%$ and $43 \%$, respectively $(4,5)$. Computed tomography (CT) and magnetic resonance imaging (MRI) are commonly used methods for detecting disease recurrence after hepatectomy, but they have the disadvantage of being expensive, and are therefore unavailable in many hospitals. They also lack sensitivity and specificity for the early detection of recurrence.

$\alpha$-Fetoprotein (AFP) is an embryo protein produced by the yolk sac and fetal liver during fetal development. It is 
the most widely used tumor biomarker currently available for the detection of liver cancer $(6,7)$, and is therefore often used to diagnose and monitor liver cancer in patients $(8,9)$. It has been shown that $60-70 \%$ of patients with liver cancer, especially hepatocellular carcinoma (HCC), have elevated AFP levels, which are lower in patients with intrahepatic cholangiocarcinoma (ICC) and mixed-type HCC $(10,11)$. AFP for liver cancer diagnosis currently has unsatisfactory sensitivity in clinical practice (12), and previously published studies have demonstrated that higher AFP levels result in worse overall survival (OS) in HCC patients after hepatectomy $(12,13)$, whereas its effects on the prognosis of other histological types of liver cancer (ICC or mixed-type HCC) are not clear. Moreover, it has been shown that normal AFP levels have low sensitivity and specificity for monitoring recurrence (13), especially in preoperative AFP-negative liver cancer (14). A previously published study showed that there was an upward trend in AFP levels during liver cancer recurrence (15). The results suggest that a slight rise in serum AFP may be sensitive for detecting the recurrence of liver cancer. In another study, elevated carcinoembryonic antigen (CEA) was found to be highly sensitive and specific for predicting the recurrence of colorectal cancer after surgery (16). The results showed that changes in traditional tumor biomarkers, such as CEA or AFP, are potentially useful for monitoring tumor recurrence with high sensitivity and specificity. However, the effects of serum delta AFP $(\triangle \mathrm{AFP})$ on the detection of treatment outcomes and on the prediction of prognosis in HCC patients after hepatectomy are unknown.

In the present study, we evaluated the effects of $\triangle \mathrm{AFP}$ on detecting recurrence and predicting prognosis in patients with liver cancer treated with hepatectomy and without distant metastasis. We present the following article in accordance with the STROBE reporting checklist (available at http://dx.doi.org/10.21037/tcr-20-1874).

\section{Methods}

\section{Patient enrollment}

We retrospectively analyzed patients who underwent liver tumor resection without lymph node or distant metastasis at Sun Yat-sen University Cancer Center between January 2012 and September 2017. The inclusion criteria were a diagnosis of liver cancer and the availability of complete clinical and follow-up data. Data collected included sex, age, smoking history, history of alcohol consumption, Child-
Pugh score, model for end-stage liver disease (MELD) score, albumin-to-bilirubin (ALBI) score, tumor-nodemetastasis (TNM) stage, tumor size, tumor differentiation, vascular invasion, tumor multiplicity, neural invasion, hepatitis virus infection, liver cirrhosis, preoperative AFP level, postoperative AFP level, surgery date, recurrence date, and last follow-up date. The Child-Pugh, MELD, and ALBI scores according to formulas (17-19). The TNM stage in the present study was defined according to the AJCC Cancer Staging Manual, 8th edition, for liver cancer. The exclusion criteria were relapse-free patients re-examined after hepatectomy fewer than two times, tumor recurrence at the first re-examination after hepatectomy, diagnosis with secondary primary cancer, and lymph node metastasis or organ invasion found by pathological examination. Patients' baseline characteristics are summarized in Table 1. The present study was approved by the Clinical Research Ethics Committee of Sun Yat-sen University Cancer Center (No. B2019-019-01). All procedures were strictly in accordance with the appropriate version of the Declaration of Helsinki (as revised in Brazil 2013). Individual consent for this retrospective analysis was waived.

\section{Laboratory measurements}

The AFP level was tested with the collected serum samples that were stored at room temperature and centrifuged at $3,500 \times \mathrm{g} / \mathrm{min}$ for 8 minutes. A Roche E-170 automatic electrochemistry analyzer was used to measure the lipid level.

\section{Follow-up}

After curative hepatectomy, patients underwent followup and examinations, including AFP serum test, CT scan, and MRI, in the first month after surgery and then every 3 months during the first 2 years after hepatectomy and every 6 months thereafter. Recurrence was determined by CT, MRI, and medical records. The research end-points were disease-free survival (DFS) and OS.

\section{Statistical analysis}

Statistical analyses were performed using SPSS version 20.0. Pearson's $\chi^{2}$-test or Fisher's exact test was chosen for evaluating the correlation between liver cancer recurrence and the clinical characteristics and pathological results. Survival curves were constructed using the Kaplan-Meier 
Table 1 Association of clinical features with liver cancer recurrence

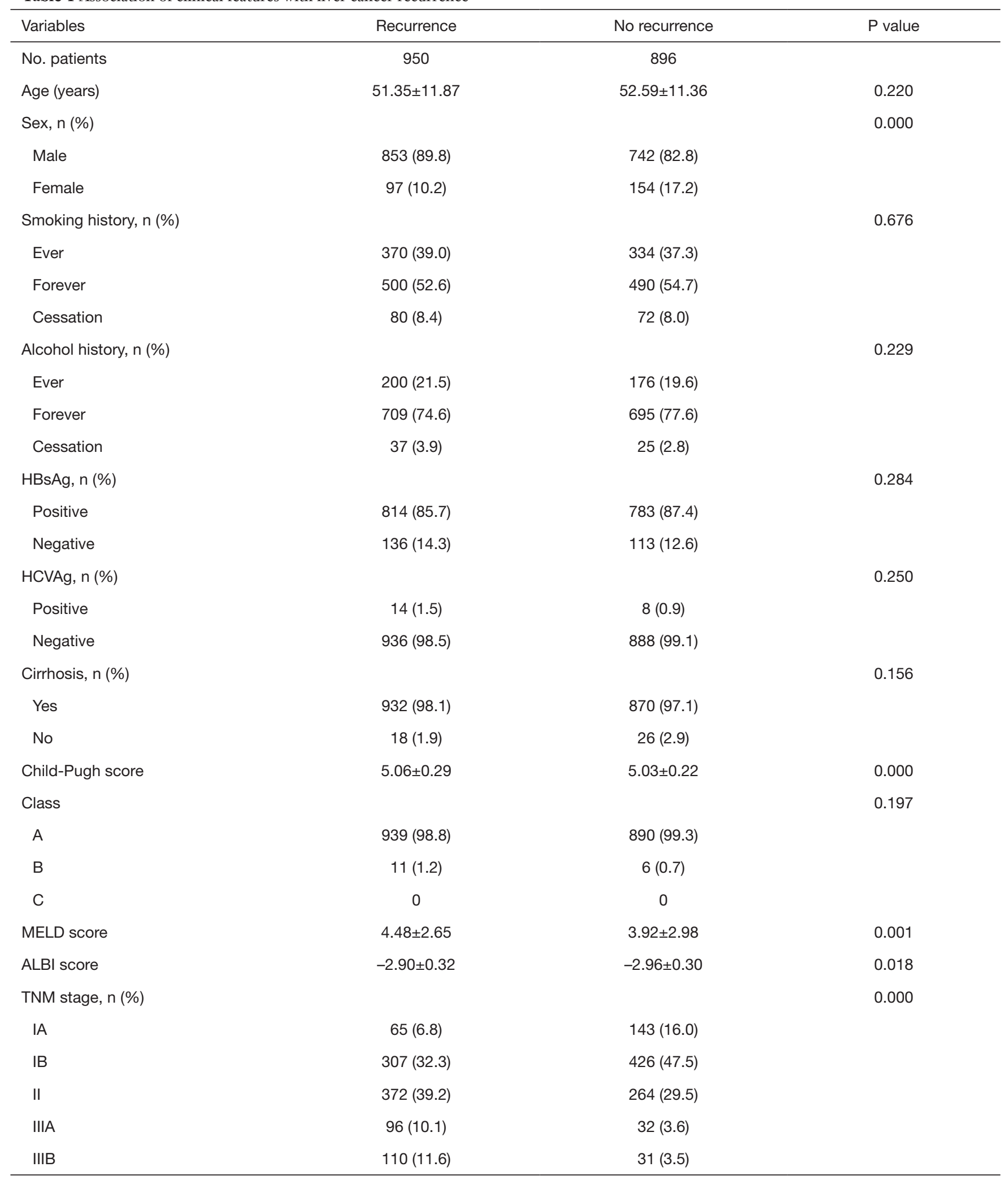

Table 1 (continued) 
Table 1 (continued)

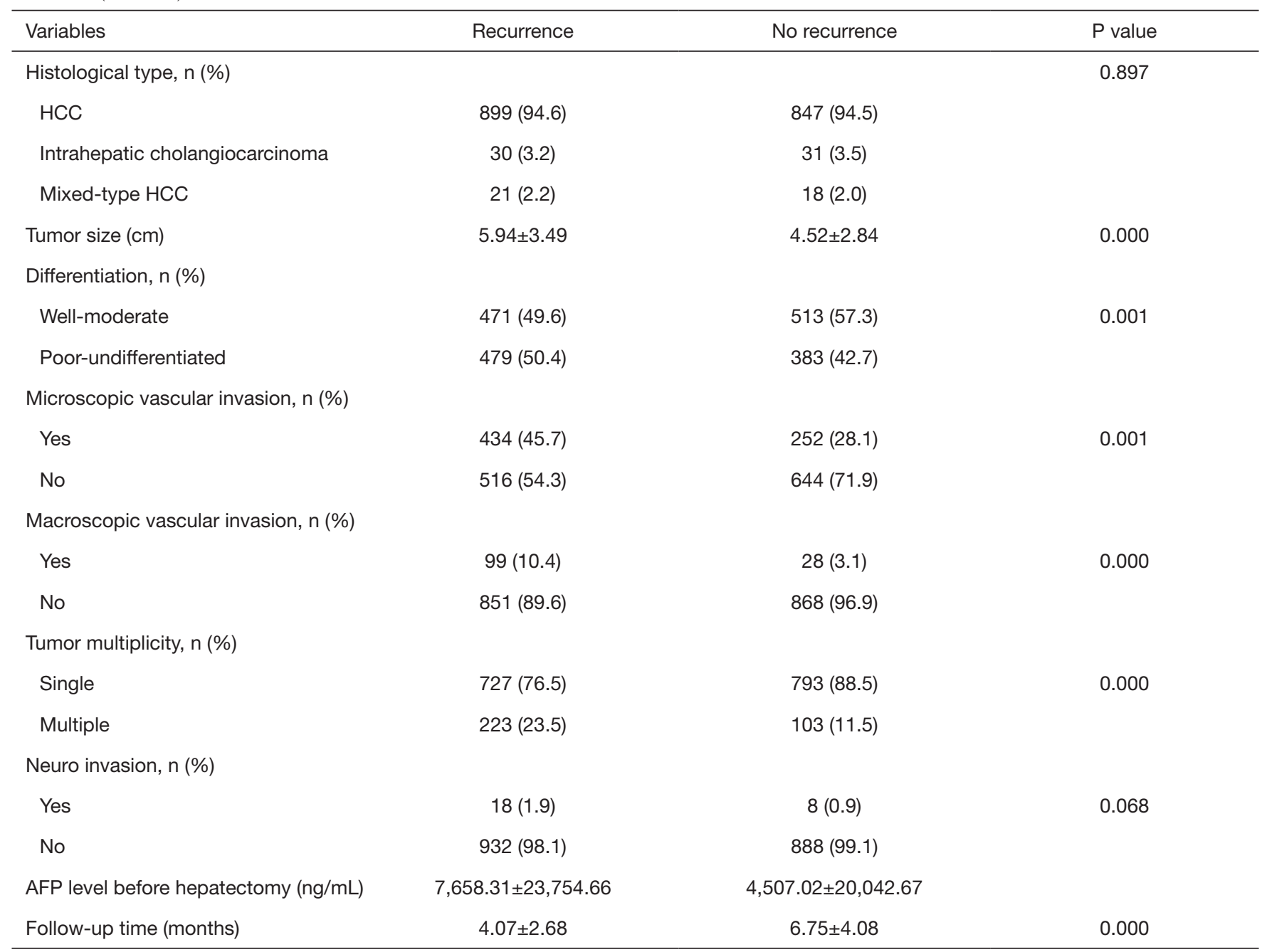

AFP, $\alpha$-fetoprotein; HBsAg, hepatitis B virus surface antigen; HCVAg, hepatitis C virus antigen; MELD, model for end-stage liver disease; ALBI, albumin-to-bilirubin.

method and log-rank test. $\triangle \mathrm{AFP}$ was equal to $\mathrm{AFP}$ at current re-examination minus AFP at the last re-examination after hepatectomy. Receiver operating characteristic (ROC) curve analysis was used to determine the cutoff value of the $\triangle \mathrm{AFP}$ level. Comparisons of sensitivity and specificity of AFP and $\triangle \mathrm{AFP}$ were analyzed using Pearson's $\chi^{2}$-test. $\mathrm{P}<0.05$ was considered statistically significant.

\section{Results}

\section{Patients' clinical characteristics}

A total of 1,846 patients who underwent hepatectomy were enrolled in the present study. Of these, $51.5 \%(950 / 1,846)$ had experienced tumor recurrence by the last follow-up. The median follow-up time was 31 months (range, 1.279.5 months). The relationship between tumor recurrence and patients' clinical characteristics is summarized in Table 1 . Tumor recurrence was correlated with male sex, higher Child-Pugh score, higher MELD score, higher ALBI score, higher TNM stage, larger tumor size, poorly differentiated tumors, microscopic vascular invasion, macroscopic vascular invasion, and tumor multiplicity $(\mathrm{P}<0.05)$ (Table 1). Age, smoking history, history of alcohol consumption, hepatitis $B$ virus (HBV) infection, hepatitis $\mathrm{C}$ virus $(\mathrm{HCV})$ infection, liver cirrhosis, histological type, and neural invasion were not statistically different between patients with and without recurrence $(\mathrm{P}>0.05)$. 
A

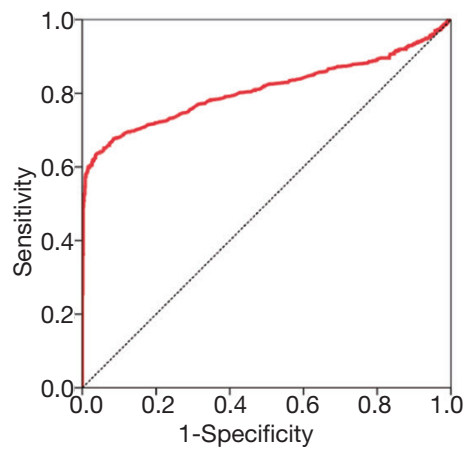

B

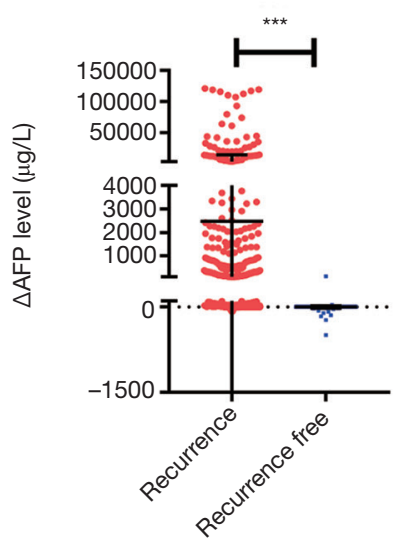

C

\begin{tabular}{|c|c|c|c|}
\hline All cases & $\begin{array}{c}\text { Real } \\
\text { recurrence }\end{array}$ & $\begin{array}{c}\text { Real no } \\
\text { recurrence }\end{array}$ & \\
\hline Predict recurrence & 568 & 14 & 582 \\
\hline Predict unrecurrence & 382 & 886 & 1264 \\
\hline Total & 950 & 896 & 1846 \\
\hline Correct & 568 & 886 & 1454 \\
\hline Sensitivity (\%) & $\mathbf{5 9 . 8}$ & & \\
\hline Specificity (\%) & & $\mathbf{9 8 . 4}$ & \\
\hline
\end{tabular}

E

\begin{tabular}{|c|c|c|c|}
\hline HCC & $\begin{array}{c}\text { Real } \\
\text { recurrence }\end{array}$ & $\begin{array}{c}\text { Real no } \\
\text { recurrence }\end{array}$ & \\
\hline Predict recurrence & 543 & 14 & 557 \\
\hline Predict unrecurrence & 356 & 833 & 1189 \\
\hline Total & 899 & 847 & 1746 \\
\hline Correct & 543 & 833 & 1376 \\
\hline Sensitivity (\%) & $\mathbf{6 0 . 4}$ & & \\
\hline Specificity (\%) & & $\mathbf{9 8 . 3}$ & \\
\hline
\end{tabular}

G

\begin{tabular}{|c|c|c|c|}
\hline ICC & $\begin{array}{c}\text { Real } \\
\text { recurrence }\end{array}$ & $\begin{array}{c}\text { Real no } \\
\text { recurrence }\end{array}$ & \\
\hline Predict recurrence & 11 & 0 & 11 \\
\hline Predict unrecurrence & 19 & 31 & 50 \\
\hline Total & 30 & 31 & 61 \\
\hline Correct & 11 & 31 & 42 \\
\hline Sensitivity (\%) & $\mathbf{3 6 . 7}$ & & \\
\hline Specificity (\%) & & $\mathbf{1 0 0}$ & \\
\hline
\end{tabular}

I

\begin{tabular}{|c|c|c|c|}
\hline Mix-type HCC & $\begin{array}{c}\text { Real } \\
\text { recurrence }\end{array}$ & $\begin{array}{c}\text { Real no } \\
\text { recurrence }\end{array}$ & \\
\hline Predict recurrence & 14 & 0 & 14 \\
\hline Predict unrecurrence & 7 & 18 & 25 \\
\hline Total & 21 & 18 & 39 \\
\hline Correct & 14 & 18 & 32 \\
\hline Sensitivity (\%) & $\mathbf{6 6 . 7}$ & & \\
\hline Specificity (\%) & & $\mathbf{1 0 0}$ & \\
\hline
\end{tabular}

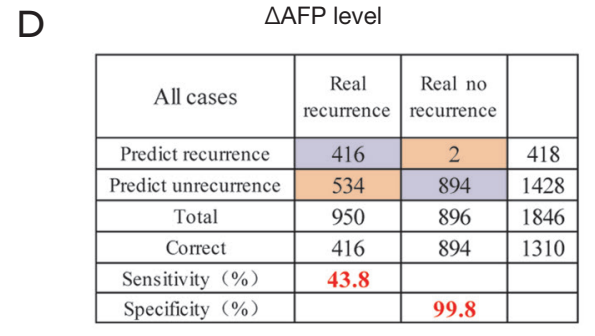

F

\begin{tabular}{|c|c|c|c|}
\hline HCC & $\begin{array}{c}\text { Real } \\
\text { recurrence }\end{array}$ & $\begin{array}{c}\text { Real no } \\
\text { recurrence }\end{array}$ & \\
\hline Predict recurrence & 401 & 2 & 403 \\
\hline Predict unrecurrence & 498 & 845 & 1343 \\
\hline Total & 899 & 847 & 1746 \\
\hline Correct & 401 & 845 & 1246 \\
\hline Sensitivity (\%) & 44.6 & & \\
\hline Specificity (\%) & & 99.8 & \\
\hline
\end{tabular}

$\mathrm{H}$

\begin{tabular}{|c|c|c|c|}
\hline ICC & $\begin{array}{c}\text { Real } \\
\text { recurrence }\end{array}$ & $\begin{array}{c}\text { Real no } \\
\text { recurrence }\end{array}$ & \\
\hline Predict recurrence & 2 & 0 & 2 \\
\hline Predict unrecurrence & 28 & 31 & 59 \\
\hline Total & 30 & 31 & 61 \\
\hline Correct & 2 & 31 & 33 \\
\hline Sensitivity (\%) & $\mathbf{6 . 7}$ & & \\
\hline Specificity (\%) & & $\mathbf{1 0 0}$ & \\
\hline
\end{tabular}

J

\begin{tabular}{|c|c|c|c|}
\hline Mix-type HCC & $\begin{array}{c}\text { Real } \\
\text { recurrence }\end{array}$ & $\begin{array}{c}\text { Real no } \\
\text { recurrence }\end{array}$ & \\
\hline Predict recurrence & 13 & 0 & 13 \\
\hline Predict unrecurrence & 8 & 18 & 26 \\
\hline Total & 21 & 18 & 39 \\
\hline Correct & 13 & 18 & 31 \\
\hline Sensitivity (\%) & $\mathbf{6 1 . 9}$ & & \\
\hline Specificity (\%) & & $\mathbf{1 0 0}$ & \\
\hline
\end{tabular}

Figure 1 Delta $\alpha$-fetoprotein $(\triangle \mathrm{AFP})$ cutoff level was compared to normal AFP level to detect recurrence (A). $\triangle \mathrm{AFP}$ cutoff value was obtained from the receiver operating characteristic curve. $\triangle \mathrm{AFP}$ was significantly higher in patients with than without recurrence at last follow-up (B). $\triangle \mathrm{AFP}$ level was used to detect tumor recurrence in liver cancer (C). $\triangle \mathrm{AFP}$ level in hepatocellular carcinoma (HCC) (E), intrahepatic cholangiocarcinoma (ICC) (G), and mixed-type HCC (I) was more sensitive than AFP level in liver cancer (D); HCC (F), ICC $(\mathrm{H})$, and mixed-type HCC (J). *** ${ }^{*}<0.001$. AFP, $\alpha$-fetoprotein; HCC, hepatocellular carcinoma; ICC, intrahepatic cholangiocarcinoma.

\section{$\triangle A F P$ in the detection of liver cancer recurrence}

The $\triangle$ AFP cutoff value of $1.295 \mathrm{ng} / \mathrm{mL}$ for detecting recurrence was generated using $\mathrm{ROC}$ curve analysis (Figure $1 A$ ). The $\triangle \mathrm{AFP}$ level was significantly higher in patients with tumor recurrence than in those without $(\mathrm{P}<0.05)$ (Figure 1B). There was no difference between normal AFP and $\triangle \mathrm{AFP}$ at recurrence or last followup regarding sex, smoking history, history of alcohol consumption, HBV surface antigen, $\mathrm{HCV}$ antigen, cirrhosis, or histology type (Table 2). However, there was a significant difference between normal and $\triangle \mathrm{AFP}$ levels in patients with recurrent liver cancer (Table 3). Compared to
AFP in the normal range, $\triangle \mathrm{AFP}$ showed more sensitivity (59.8\% vs. $43.8 \%$ ), but similar specificity (98.4\% vs. $99.8 \%$ ) for monitoring liver cancer recurrence after liver resection. In the three main histological subtypes of liver cancer, $\triangle \mathrm{AFP}$ also showed more sensitivity and similar specificity as $\mathrm{AFP}$ (Figure $1 C, D, E, F, G, H, I, 7$ ). $\triangle \mathrm{AFP}$ was more sensitive than normal AFP in both preoperative AFP-positive and preoperative AFP-negative patients. In AFP-positive patients, $\triangle \mathrm{AFP}$ was more sensitive than normal AFP (77.16\% vs. $63.28 \%)$, and specificity was similar $(98.30 \%$ vs. 99.58\%); the area under the curve (AUC) of the ROC curve was 0.877 and 0.814 , respectively. In AFP-negative patients, $\triangle \mathrm{AFP}$ was more sensitive than normal AFP 
Table $2 \alpha$-Fetoprotein (AFP) and delta AFP ( $\triangle \mathrm{AFP}$ ) levels associated with clinical characteristics

\begin{tabular}{|c|c|c|c|c|}
\hline Variables & AFP at recurrence/last follow-up & $P$ value & $\triangle \mathrm{AFP}$ at recurrence/last follow-up & $P$ value \\
\hline Male & $1,301.39 \pm 8,945.53$ & & $1,155.50 \pm 8,373.60$ & \\
\hline Female & $4,463.75 \pm 42,092.62$ & & $4,305.16 \pm 41,695.66$ & \\
\hline Smoking history (ng/mL) & & 0.746 & & 0.738 \\
\hline Forever & $1,801.14 \pm 22,105.20$ & & $1,651.22 \pm 21,783.53$ & \\
\hline Cessation & $686.50 \pm 3,614.10$ & & $649.27 \pm 3,551.21$ & \\
\hline Alcohol history (ng/mL) & & 0.806 & & 0.836 \\
\hline Ever & $2,179.41 \pm 12,949.95$ & & $1,989.78 \pm 12,164.40$ & \\
\hline HBsAg (ng/mL) & & 0.404 & & 0.423 \\
\hline Positive & $1,438.97 \pm 10,006.45$ & & $1,304.80 \pm 9,415.4$ & \\
\hline Negative & $3,606.73 \pm 40,751.70$ & & $3,372.93 \pm 40,468.18$ & \\
\hline HCVAg (ng/mL) & & 0.667 & & 0.681 \\
\hline Positive & $175.47 \pm 706.13$ & & $79.35 \pm 299.10$ & \\
\hline Negative & $1,750.14 \pm 17,722.92$ & & $1,601.90 \pm 17,345.79$ & \\
\hline Cirrhosis (ng/mL) & & 0.853 & & 0.821 \\
\hline Yes & $1,719.51 \pm 17,728.82$ & & $1,569.53 \pm 17,350.87$ & \\
\hline
\end{tabular}

HBsAg, hepatitis B virus surface antigen; HCC, hepatocellular carcinoma; HCVAg, hepatitis C virus antigen; ICC, intrahepatic cholangiocarcinoma.

Table 3 A-Fetoprotein (AFP) and delta AFP ( $\triangle \mathrm{AFP}$ ) levels associated with recurrence and clinical characteristic

\begin{tabular}{lccc}
\hline Variables & AFP level & P value & $\Delta$ AFP level \\
\hline At recurrence $(\mathrm{ng} / \mathrm{mL})$ & $3,361.29 \pm 24,453.40$ & 0.000 & $3,079.15 \pm 23,946.13$ \\
At last follow-up (no recurrence) $(\mathrm{ng} / \mathrm{mL})$ & $3.23 \pm 5.64$ & & $-1.76 \pm 20.98$ \\
Type of recurrence $(\mathrm{ng} / \mathrm{mL})$ & & 0.000 & 0.000 \\
Intrahepatic recurrence only $(\mathrm{n}=767)$ & $2,242.85 \pm 12,052.48$ & & $1,993.86 \pm 11,264.97$ \\
Intra- and extrahepatic recurrence $(\mathrm{n}=63)$ & $11,449.77 \pm 28,982.30$ & $10,626.11 \pm 27,260.61$ \\
Extrahepatic recurrence only $(\mathrm{n}=120)$ & $6,263.55 \pm 57,834.24$ & $6,053.75 \pm 57,638.38$ \\
\hline
\end{tabular}


(31.20\% vs. $11.70 \%)$, and specificity was similar (99.03\% vs. $100.00 \%$ ); the AUC of the ROC curve was 0.649 and 0.558 , respectively (Figure $2 A, B$ ).

$\triangle \mathrm{AFP}$ performed well for monitoring recurrence for liver cancer patients with early and late recurrence after resection. Early recurrence refers to recurrence within 1 year after hepatectomy, and late recurrence refers to recurrence $>1$ year after hepatectomy. The results showed that $\triangle \mathrm{AFP}$ could predict $78.13 \%(125 / 160)$ and $56.08 \%$ (443/790) of early and late liver cancer recurrence, respectively. In comparison, AFP was found to predict $63.75 \%(102 / 160)$ and $39.75 \%(314 / 790)$ of early and late liver cancer recurrence, respectively $(\mathrm{P}<0.05)$.

\section{$\triangle A F P$ showed a low false-positive rate when predicting recurrence}

To validate the false-positive rate of the $\triangle \mathrm{AFP}$ cutoff value during follow-up, we measured $\triangle \mathrm{AFP}$ levels in recurrencefree patients $(896 / 1,846)$, who had an average of $6.75 \pm 4.08$ re-examinations after hepatectomy. The false-positive rates were $2.86 \%, 2.90 \%, 1.49 \%$, and $4.13 \%$ for liver cancer, HCC, ICC, and mixed-type HCC, respectively (Figure 2C). However, 174 of 950 patients with recurrence exhibited $\triangle \mathrm{AFP}$ exceeding the cutoff value before the clinical diagnosis of tumor recurrence, as determined by CT or MRI. In these patients, the time predicted by $\triangle \mathrm{AFP}$ was 158.33 days for liver cancer, 159.25 days for HCC, 124.50 days for ICC, and 149.33 days for mixed-type HCC compared with CT or MRI (Figure 2D).

\section{$\triangle A F P$ and liver cancer prognosis after bepatectomy}

We analyzed the effect on prognosis between patients who exceeded the $\triangle \mathrm{AFP}$ cutoff value more than once (exceeded group) and those who never exceeded the cutoff value (never-exceeded group) after hepatectomy. The median DFS for the never-exceeded and exceeded groups was $74.71 \pm 8.01$ and $11.27 \pm 0.62$ months, respectively. Kaplan-Meier survival analysis showed that liver cancer patients who never exceeded the $\triangle \mathrm{AFP}$ cut-off value after hepatectomy had a longer DFS $(\mathrm{P}<0.001)$ and OS $(\mathrm{P}<0.001)$ than those who exceeded the cutoff value more than once (Figure 3A,B). The subtype analysis of patients by histological type showed better DFS in the neverexceeded group compared with the exceeded group (HCC group: $\mathrm{P}<0.001$; ICC group: $\mathrm{P}=0.007$; mixed-type HCC: $\mathrm{P}=0.007)$. OS was significantly different between the never- exceeded and exceeded groups in $\mathrm{HCC}$ patients $(\mathrm{P}<0.001)$, but not in ICC $(\mathrm{P}=0.365)$ and mixed-type HCC $(\mathrm{P}=0.649)$ patients (Figure $3 C, D, E, F, G, H$ ). In the Cox regression analyses, the adjusted hazard ratios (HRs) for DFS revealed significant differences between the exceeded and neverexceeded group in all cases [HR: 4.209, 95\% confidence interval (CI): 3.672-4.824, $\mathrm{P}<0.001]$ and in the three main histological types of liver cancer (HCC group, HR: 4.329, 95\% CI: 3.760-4.984, $\mathrm{P}<0.001$; ICC group, HR: 2.580, 95\% CI: 1.257-5.297, $\mathrm{P}=0.010$; mixed-type HCC group, HR: 3.403, 95\% CI: 1.316-8.800, $\mathrm{P}=0.012)$. In the survival analyses, the adjusted HRs showed a significant association with exceeding the cutoff value of $\triangle \mathrm{AFP}$ after hepatectomy in liver cancer patients (HR: 3.792, 95\% CI: 2.786-5.160, $\mathrm{P}<0.001)$. Subtype analysis of patients by histological type showed better OS in HCC patients in the never-exceeded group (HR: 4.201, 95\% CI: 2.901-5.575, $\mathrm{P}<0.001$ ), but no significant difference in ICC and mixed-type HCC patients (ICC group, HR: 1.825, 95\% CI: 0.488-6.832, P=0.372; mixed-type HCC group, HR: 1.485, 95\% CI: 0.268-8.217, $\mathrm{P}=0.651)$.

\section{Discussion}

AFP level plays an important role in liver cancer diagnosis, prognosis, recurrence monitoring, and treatment response, but the effects of $\triangle \mathrm{AFP}$ on liver cancer prognosis and surveillance are unclear. In the present study, we evaluated the ability of $\triangle \mathrm{AFP}$ in recurrence detection and prognosis based on a cutoff value. We found that a $\triangle \mathrm{AFP}$ cutoff value was more sensitive for detecting recurrence than AFP based on the normal range, and exhibited an upward trend before CT or MRI examination. However, liver cancer patients who exceeded this cutoff value more than once during follow-up had a worse prognosis after hepatectomy.

AFP level is commonly used as an indicator to monitor recurrence after hepatectomy in patients with liver cancer, especially HCC (14,20). The 2018 National Comprehensive Cancer Network Clinical Practice Guidelines in Oncology for Hepatobiliary Cancers, version 3, recommends that HCC patients after hepatectomy have serum AFP levels checked every 3 months for 2 years, and then every 6-12 months after 2 years; however, a definitive cutoff AFP value to predict recurrence and detect a change of recurrence risk level with a monitoring strategy remains unknown (21). Chang et al. reported that the best cutoff AFP value was $5.45 \mu \mathrm{g} / \mathrm{L}$ based on the last follow-up/ recurrence AFP level of $72 \mathrm{HCC}$ patients treated with 
A

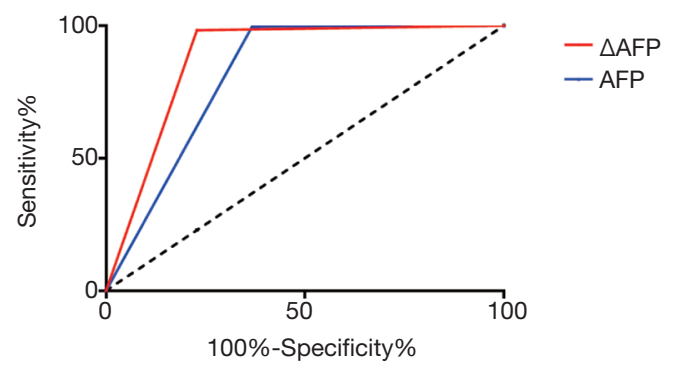

C

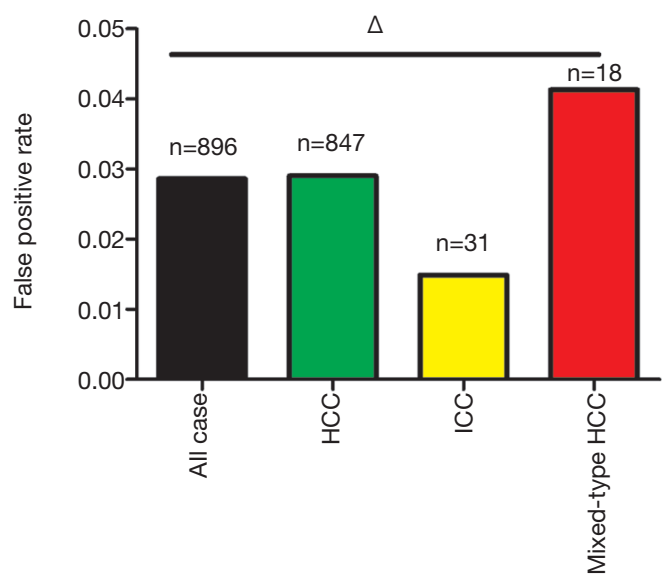

B

AFP negative

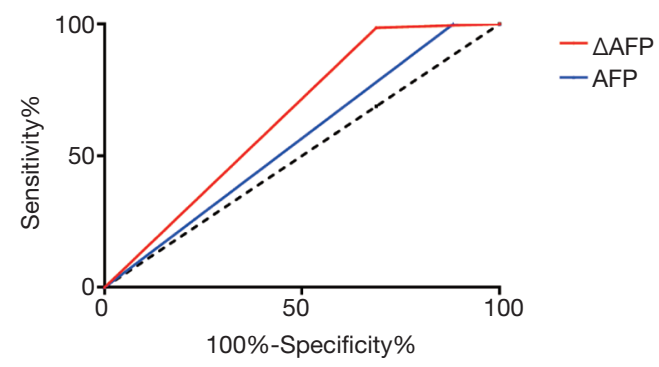

D

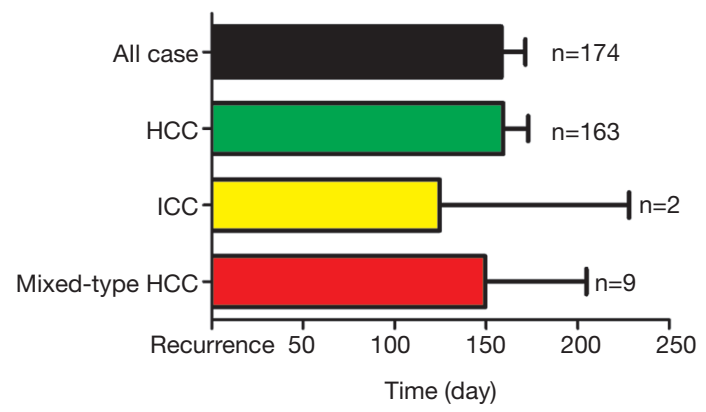

Figure 2 Subtype analyses of delta $\alpha$-fetoprotein $(\triangle \mathrm{AFP})$ cutoff value in the detection of liver cancer recurrence. $\triangle \mathrm{AFP}$ showed more sensitivity in detecting tumor recurrence in preoperative $\mathrm{AFP}$-positive (A) and AFP-negative (B) patients. $\triangle \mathrm{AFP}$ best cutoff value in patients with no recurrence showed high specificity, and each histological subtype showed no statistical difference (C). A total of 174 patients had $\triangle \mathrm{AFP}$ level exceed the cutoff value before recurrence, and the average time was earlier than that measured with computed tomography/ magnetic resonance imaging (D). $\Delta, \mathrm{P}>0.05$. AFP, $\alpha$-fetoprotein; HCC, hepatocellular carcinoma; ICC, intrahepatic cholangiocarcinoma.

hepatectomy and liver transplantation (15). This value was sensitive to AFP levels based on the normal range (84.8\% vs. $62.5 \%)$, but its specificity was lower (77.1\% vs. 94.3\%). For liver transplantation, Nörthen et al. suggested best cutoff AFP values of 7, 6, and $6 \mu \mathrm{g} / \mathrm{L}$ at 1,3 , and 5 years after liver transplantation in $63 \mathrm{HCC}$ patients with a diagnostic sensitivity of $79 \%, 81 \%$, and $77 \%$, and a specificity of $82 \%$, $79 \%$, and $69 \%$ (22). Previous studies have indicated that a cutoff value depends on AFP level after treatment with high sensitivity but low specificity, possibly due to the focus on AFP level without taking into account the variation of AFP values. Chang et al. found that some patients with recurrence exhibited an upward trend in AFP level before tumor recurrence, suggesting that the minor variable of AFP could be a sensitive biomarker in detecting liver cancer recurrence (15). However, the effect of $\triangle \mathrm{AFP}$ level on liver cancer patients after hepatectomy is not yet clearly understood. In our study, 568 of 950 patients (59.8\%) had an upward trend for the prediction of tumor recurrence. The cutoff $\triangle \mathrm{AFP}$ level was more sensitive than the AFP level based on the normal range of $0-25 \mathrm{ng} / \mathrm{mL}(59.8 \%$ vs. $43.8 \%)$, but the specificity between these values was similar (98.4\% vs. $99.5 \%)$. Our study also showed that this $\triangle \mathrm{AFP}$ cutoff level may be useful for detecting ICC and mixed-type HCC after hepatectomy. The sensitivity was $36.7 \%$ in ICC patients and $66.7 \%$ in mixed-type HCC patients, while that yielded by the normal range was $6.7 \%$ and $61.9 \%$, respectively. Specificity was $100 \%$ for all histological types and methods. About $40 \%$ of liver cancer patients were AFP-negative preoperatively, and had different biologic characteristics than those with AFP-positive liver cancer (23). Previous studies have found that AFP-positive liver cancer is associated with a worse prognosis compared with AFP-negative liver cancer (24). However, in current 
A

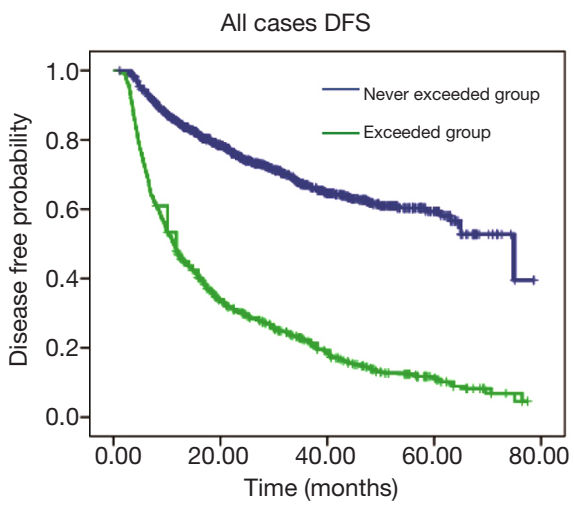

B

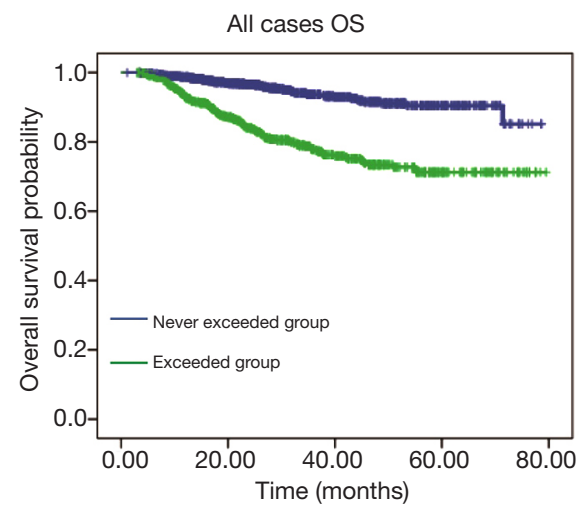

C

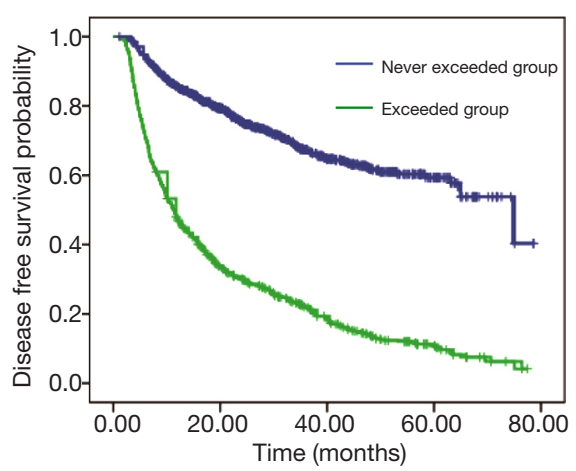

$\mathrm{F}$

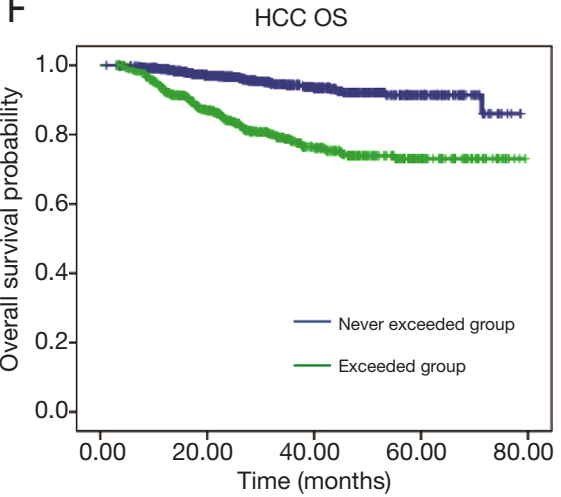

$\mathrm{D}$

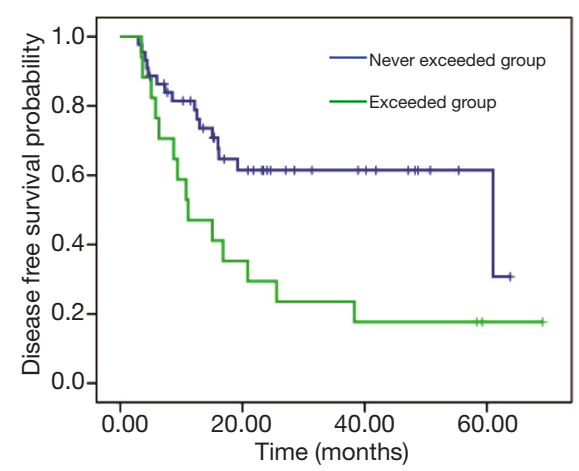

G

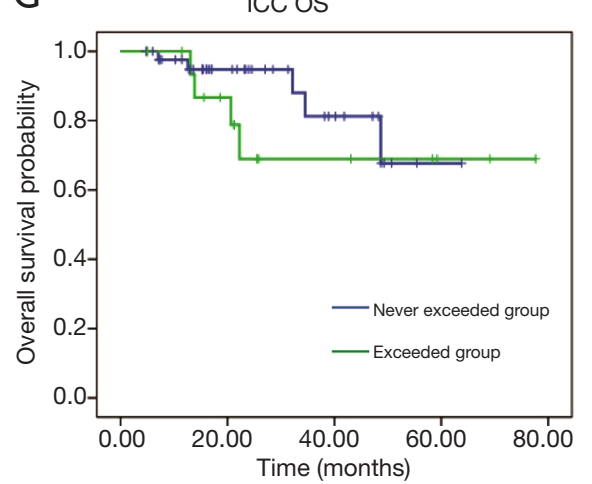

$\mathrm{E}$

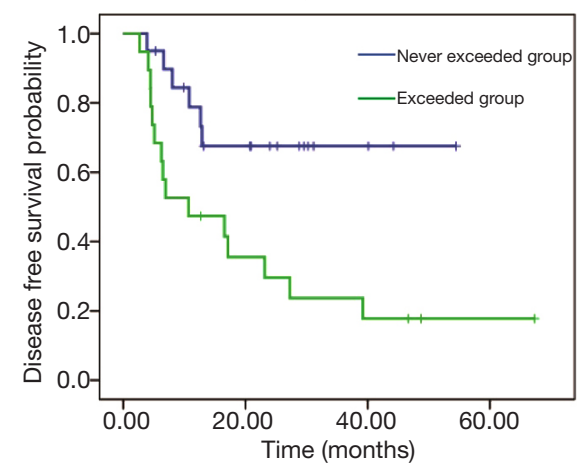

$\mathrm{H}$

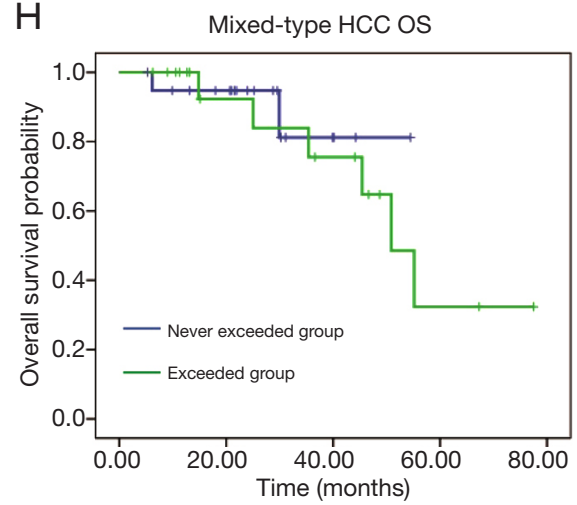

Figure 3 Delta $\alpha$-fetoprotein $(\triangle \mathrm{AFP})$ level predicts prognosis in hepatocellular carcinoma (HCC) patients. Kaplan-Meier analyses showed differences in recurrence probabilities among all cases. $(A, C, D, E)$ Liver cancer $(P<0.001)(A), H C C(P<0.001)(C)$, intrahepatic cholangiocarcinoma (ICC) $(\mathrm{P}=0.007)(\mathrm{D})$, and mixed-type HCC $(\mathrm{P}=0.007)(\mathrm{E})$ patients that exceeded the cutoff value more than once and those that never exceeded the value. Overall survival probabilities between liver cancer $(\mathrm{P}<0.001)(\mathrm{B})$ and HCC $(\mathrm{P}<0.001)(\mathrm{F})$ showed significant difference between the exceeded and never-exceeded groups, but ICC ( $\mathrm{P}=0.365)(\mathrm{G})$ and mixed-type HCC $(\mathrm{P}=0.649)(\mathrm{H})$ showed no difference between the two groups. AFP, $\alpha$-fetoprotein. 
clinical practice, there is a lack of appropriate indicators for detecting recurrence of liver cancer after hepatectomy among liver cancer patients. Our findings suggest that $\triangle \mathrm{AFP}$ is more sensitive than $\mathrm{AFP}$ in detecting recurrence among AFP-negative patients (31.2\% vs. $11.7 \%$ ). In addition, the $\triangle \mathrm{AFP}$ cut-off level showed high specificity in 896 recurrence-free patients. The false-positive result might have been due to benign conditions after surgery, such as liver inflammation and cirrhosis (25). However, we found that 174 of 950 patients with recurrence had exceeded $\triangle \mathrm{AFP}$ cutoff levels before recurrence was detected by MRI or CT scan, which likely means that tumor recurrence was below the level of detection on CT or MRI, but which might have led to increased AFP levels. These data suggest that the $\triangle \mathrm{AFP}$ cutoff level may not only be used for detecting liver cancer postoperatively, but could also have potential for the early prediction of recurrence after hepatectomy (26).

In the survival analysis, we found that liver cancer patients who had exceeded $\triangle \mathrm{AFP}$ cut-off levels during follow-up exhibited worse DFS and OS than patients who had never exceeded $\triangle \mathrm{AFP}$ cutoff levels. This finding suggests that an exceeded $\triangle \mathrm{AFP}$ cut-off level during follow-up is a prognostic indicator of liver cancer after hepatectomy. There was no difference in OS between the exceeded group and never-exceeded group in ICC and mixed-type HCC patients, but there was a significant difference in HCC patients. One reason for this difference may be due to the small sample size of patients with ICC and mixed-type HCC. Another reason may be that ICC and mixed-type HCC express lower levels of AFP than HCC $(11,27,28)$. Several studies have reported that preoperative AFP level is a prognostic indicator in HCC patients (27-30). Although previous studies have reported that changes in AFP levels between pre- and post-hepatectomy can predict surgical outcomes $(14,31,32)$, the effects of variations in AFP after hepatectomy remain unclear. The results of our study provide a new indicator for the real-time monitoring of recurrence and the evaluation of the prognosis of liver cancer after hepatectomy. If the $\triangle \mathrm{AFP}$ exceeds the cutoff value during the postoperative monitoring of liver cancer patients, it is suggested that the frequency of re-examination is increased, or that additional examinations, such as chest CT or isotope bone scan, are undertaken for the early detection of metastatic and recurrent lesions to improve the prognosis of liver cancer.

The results of the present study showed that the $\Delta \mathrm{AFP}$ cutoff level was sufficiently sensitive for monitoring recurrence after liver cancer hepatectomy, and patients with recurrence exhibited an upward trend with $\triangle \mathrm{AFP}$ compared to MRI. However, the present study has some limitations. We excluded patients with metastasis and recurrence 1 month after surgery; therefore, subsequent studies are needed to validate and explore the $\triangle \mathrm{AFP}$ cutoff level in all liver cancer patients, including those with inoperable cancer. In addition, as a retrospective study, we were not able to monitor AFP levels in a strict manner with regular frequency, which may have affected the observed fluctuations in AFP level. Finally, different institutions may have different values for the normal range. Therefore, further prospective studies are required to further explore and validate the detection ability of the $\triangle \mathrm{AFP}$ cutoff level.

\section{Acknowledgments}

Funding: None.

\section{Footnote}

Reporting Checklist: The authors have completed the STROBE reporting checklist. Available at http://dx.doi. org/10.21037/tcr-20-1874

Data Sharing Statement: Available at http://dx.doi. org/10.21037/tcr-20-1874

Peer Review File: Available at http://dx.doi.org/10.21037/tcr20-1874

Conflicts of Interest: All authors have completed the ICMJE uniform disclosure form (available at http://dx.doi. org/10.21037/tcr-20-1874). The authors have no conflicts of interest to declare.

Ethical Statement: The authors are accountable for all aspects of the work in ensuring that questions related to the accuracy or integrity of any part of the work are appropriately investigated and resolved. The present study was approved by the Clinical Research Ethics Committee of Sun Yat-sen University Cancer Center (no. B2019019-01). All procedures were strictly in accordance with the appropriate version of the Declaration of Helsinki (as revised in Brazil 2013). Individual consent for this retrospective analysis was waived.

Open Access Statement: This is an Open Access article distributed in accordance with the Creative Commons 
Attribution-NonCommercial-NoDerivs 4.0 International License (CC BY-NC-ND 4.0), which permits the noncommercial replication and distribution of the article with the strict proviso that no changes or edits are made and the original work is properly cited (including links to both the formal publication through the relevant DOI and the license). See: https://creativecommons.org/licenses/by-nc-nd/4.0/.

\section{References}

1. Fu J, Wang H. Precision diagnosis and treatment of liver cancer in China. Cancer Lett 2018;412:283-8.

2. Chang KV, Chen JD, Wu WT, et al. Association between Loss of Skeletal Muscle Mass and Mortality and Tumor Recurrence in Hepatocellular Carcinoma: A Systematic Review and Meta-Analysis. Liver Cancer 2018;7:90-103.

3. Xiao Y, Li W, Wan H, et al. Central hepatectomy versus major hepatectomy for patients with centrally located hepatocellular carcinoma: A meta-analysis. Int J Surg 2018;52:297-302.

4. Mise Y, Hasegawa K, Shindoh J, et al. The Feasibility of Third or More Repeat Hepatectomy for Recurrent Hepatocellular Carcinoma. Ann Surg 2015;262:347-57.

5. Yamashita Y, Shirabe K, Tsuijita E, et al. Third or more repeat hepatectomy for recurrent hepatocellular carcinoma. Surgery 2013;154:1038-45.

6. Nanashima A, Taura N, Abo T, et al. Tumor marker levels before and after curative treatment of hepatocellular carcinoma as predictors of patient survival. Dig Dis Sci 2011;56:3086-100.

7. Marrero JA, Feng Z, Wang Y, et al. Alpha-fetoprotein, des-gamma carboxyprothrombin, and lectin-bound alpha-fetoprotein in early hepatocellular carcinoma. Gastroenterology 2009;137:110-8.

8. Toro A, Ardiri A, Mannino M, et al. Effect of pre- and post-treatment $\alpha$-fetoprotein levels and tumor size on survival of patients with hepatocellular carcinoma treated by resection, transarterial chemoembolization or radiofrequency ablation: a retrospective study. BMC Surg 2014;14:40.

9. Ma WJ, Wang HY, Teng LS. Correlation analysis of preoperative serum alpha-fetoprotein (AFP) level and prognosis of hepatocellular carcinoma (HCC) after hepatectomy. World J Surg Oncol 2013;11:212.

10. Marrero JA, Kulik LM, Sirlin CB, et al. Diagnosis, Staging, and Management of Hepatocellular Carcinoma: 2018 Practice Guidance by the American Association for the Study of Liver Diseases. Hepatology 2018;68:723-50.
11. Yap AQ, Chen CL, Yong CC, et al. Clinicopathological factors impact the survival outcome following the resection of combined hepatocellular carcinoma and cholangiocarcinoma. Surg Oncol 2013;22:55-60.

12. Farinati F, Marino D, De Giorgio M, et al. Diagnostic and prognostic role of alpha-fetoprotein in hepatocellular carcinoma: both or neither. Am J Gastroenterol 2006;101:524-32.

13. Chon YE, Choi GH, Lee MH, et al. Combined measurement of preoperative $\alpha$-fetoprotein and des- $\gamma$ carboxy prothrombin predicts recurrence after curative resection in patients with hepatitis-B-related hepatocellular carcinoma. Int J Cancer 2012;131:2332-41.

14. Nobuoka D, Kato Y, Gotohda N, et al. Postoperative serum alpha-fetoprotein level is a useful predictor of recurrence after hepatectomy for hepatocellular carcinoma. Oncol Rep 2010;24:521-8.

15. Chang SK, Hlaing WW, Yu RQ, et al. Value of alphafoetoprotein for screening of recurrence in hepatocellular carcinoma post resection. Singapore Med J 2012;53:32-5.

16. Litvak A, Cercek A, Segal N, et al. False-positive elevations of carcinoembryonic antigen in patients with a history of resected colorectal cancer. J Natl Compr Canc Netw 2014;12:907-13.

17. Pugh RN, Murray-Lyon IM, Dawson JL, et al. Transection of the oesophagus for bleeding oesophageal varices. $\mathrm{Br} \mathrm{J}$ Surg 1973;60:646-9.

18. Kamath PS, Kim WR. The model for end-stage liver disease (MELD). Hepatology 2007;45:797-805.

19. Johnson PJ, Berhane S, Kagebayashi C, et al. Assessment of liver function in patients with hepatocellular carcinoma: a new evidence-based approach-the ALBI grade. J Clin Oncol 2015;33:550-8

20. Siripongsakun S, Wei SH, Lin S, et al. Evaluation of alpha-fetoprotein in detecting hepatocellular carcinoma recurrence after radiofrequency ablation. J Gastroenterol Hepatol 2014;29:157-64.

21. NCCN Clinical Practice Guidelines in Oncology, Hepatobiliary cancers, Version 3. 2018.

22. Nörthen A, Asendorf T, Walson PD, et al. Diagnostic value of alpha-1-fetoprotein (AFP) as a biomarker for hepatocellular carcinoma recurrence after liver transplantation. Clin Biochem 2018;52:20-5.

23. Wang M, Devarajan K, Singal AG, et al. The Doylestown Algorithm: A Test to Improve the Performance of AFP in the Detection of Hepatocellular Carcinoma. Cancer Prev Res (Phila) 2016;9:172-9.

24. Lu Y, Zhu M, Li W, et al. Alpha fetoprotein plays a critical 
role in promoting metastasis of hepatocellular carcinoma cells. J Cell Mol Med 2016;20:549-58.

25. Kim BS, Hayashi PH, Kim SH, et al. Outcomes of patients with elevated $\alpha$-fetoprotein level and initial negative findings at MR imaging. Radiology 2013;268:109-19.

26. Li Y, Li DJ, Chen J, et al. Application of Joint Detection of AFP, CA19-9, CA125 and CEA in Identification and Diagnosis of Cholangiocarcinoma. Asian Pac J Cancer Prev 2015;16:3451-5.

27. Huang J, Wang X, Zhu Y, et al. Specific prognostic factors in hepatitis B virus-related and non-hepatitis B virusrelated intrahepatic cholangiocarcinoma after macroscopic curative resection. J Surg Oncol 2019;119:40-46.

28. Shimoda M, Tago K, Shiraki T, et al. Risk Factors for Early Recurrence of Single Lesion Hepatocellular Carcinoma

Cite this article as: Sun LY, He Y, Liu Q, Wang F. Effect of delta $\alpha$-fetoprotein on the detection of liver cancer recurrence. Transl Cancer Res 2020;9(10):6263-6274. doi: 10.21037/tcr20-1874
After Curative Resection. World J Surg 2016;40:2466-71.

29. Yang SL, Liu LP, Yang S, et al. Preoperative serum $\alpha$-fetoprotein and prognosis after hepatectomy for hepatocellular carcinoma. Br J Surg 2016;103:716-24.

30. Lee CW, Tsai HI, Sung CM, et al. Risk factors for early mortality after hepatectomy for hepatocellular carcinoma. Medicine (Baltimore) 2016;95:e5028.

31. Toyoda H, Kumada T, Tada T, et al. Changes in highly sensitive alpha-fetoprotein for the prediction of the outcome in patients with hepatocellular carcinoma after hepatectomy. Cancer Med 2014;3:643-51.

32. Rungsakulkij N, Suragul W, Mingphruedhi S, et al. Prognostic role of alpha-fetoprotein response after hepatocellular carcinoma resection. World J Clin Cases 2018;6:110-20. 